\section{Comments on the case-control study on access to health care and child mortality}

We have some comments and queries related to the paper "Access to health care and mortality of children under 5 years of age in the Gambia: a case control study" published in the Bulletin of the World Health Organization. ${ }^{1}$ We appreciate the earnest attempts of the authors in measuring the effects of non-traditional variables in addition to traditional variables. However, the instrument to measure the social support variables shows vast overlapping, with such options as "someone who understood your problem", "showed kindness and caring" and "someone to relax". These are shown as individual variables and are heavy in both informer and observer bias. It would have been more appropriate to combine them into a complex variable. Further, "had someone to prepare meals if you were unable to" overlaps with "some showed kindness and caring".

Whereas standard textbooks in epidemiology suggest a maximum ratio of $1: 4$ between cases and controls, ${ }^{2}$ the authors have not justified choosing 1:5 for their controls. Choosing controls from the same village would have strengthened the result in terms of general availability and accessibility to transport, thus minimizing the bias. Further matching for socioeconomic status (though a tough proposition) could have strengthened the results. Since 10 controls were randomly chosen for each case before deciding the first random 5 for controls, matching for socioeconomic status could have been a distinct possibility. Similarly the village of residence could also have been matched. Describing the method of deciding the centre of a village would have been useful as well.

The study period stretches over 28 months (31 December 2003 to 30 April 2006). The extra cases and controls included for the last 4 months of data collection would modify additional recruitment in that season, the variation of which might have influenced the results.

Under causes of deaths, "fever of unknown origin" accounts for $23.3 \%$.
We feel that such a high percentage does not realistically reflect the situation. The "non-specific cause" of $24.8 \%$ makes the picture even more vague as the close identification of these causes could have given further clues on the variables measured. In total, almost half the cases are in these two categories, which is a huge deficit of information.

Table 1 in the paper says $52.9 \%$ of deaths were at home and $89.3 \%$ visited health centre or hospital. The proportion of deaths within or outside the variable of "visiting the health centre or hospital" needs further discussion.

Finally, though the authors are right that further studies are required, some specific recommendations (in conclusion) from the present study should have been made, for example, organizing community crèches to look after children when the primary caregiver is away. It is curious that a major conclusion is drawn from a reference ${ }^{3}$ rather than from the present study.

Competing interests: None declared.

\section{MB Soudarssanane ${ }^{\mathrm{a}}$ \& Dhruv K Pandey $^{\mathrm{a}}$}

\section{References}

1. Rutherford ME, Dockerty JD, Jasseh M, Howie SRC, Herbison P, Jeffries DJ, et al. Access to health care and mortality of children under 5 years of age in the Gambia: a case-control study. Bull World Health Organ 2009;87:216-24. PMID:19377718 doi:10.2471/BLT.08.052175

2. Hennekens $\mathrm{CH}$, Buring JE. Chapter 6 Casecontrol studies. In: Epidemiology in medicine first edition. Boston, MA: Little Brown and Company;1987: p.142.

3. Fantahun M, Berhane Y, Wall S, Byass P, Hogberg U. Women's involvement in household decision-making and strengthening social capital-crucial factors for child survival in Ethiopia. Acta Paediatr 2007:96:582-9. PMID:17306012 doi:10.1111/j.16512227.2007.00147.x

\section{Reconsidering global targets for tuberculosis control}

A recent paper by Dowdy \& Chaisson ${ }^{1}$ used mathematical modelling to investigate whether annual declines in tuberculosis (TB) incidence can be sustained by maintaining adequate case detection rates (CDRs). In their model, once
CDRs stabilized at any constant level below $80 \%$, the projected TB incidence also stabilized. They concluded that TB control programmes should vigorously pursue improvements in case detection, regardless of the CDRs achieved.

Performance targets for global TB control were first formulated in 1991 at the 44th World Health Assembly. National TB control programmes were encouraged to achieve CDRs of at least $70 \%$ and cure rates in excess of $85 \% .^{2}$ The underlying rationale was based on epidemiological estimates that TB prevalence, and later TB incidence, should decline by about $5-10 \%$ per year if these targets could be met and sustained. Epidemiological estimates were derived from empiric observations in European countries following the introduction of chemotherapy in the 1950 s. $^{3}$ However, even in situations where both targets were reached and achievements sustained, incidence rates failed to decline as predicted. On critical review it is evident that, even if these targets are met, only $60 \%$ $(0.7 \times 0.85=0.595)$ of TB cases will be "cured" by the programme. In addition, these targets only apply to new sputum smear-positive cases, while a huge percentage of patients in endemic areas are either retreatment cases or sputum smear-negative (particularly in HIVaffected areas).

Only two public health intervention avenues exist to gain control of the global TB epidemic. ${ }^{4}$ First, every effort should be made to reduce host vulnerability at the population level. Host vulnerability is influenced by multiple factors and creative efforts are urgently required to address issues like poverty, malnutrition and HIV infection. Second, effective measures should be implemented to limit Mycobacterium tuberculosis transmission within communities. This relates directly to the appropriateness of current WHO-defined performance targets, which is the focus of this letter. We introduce two novel concepts that have not been considered in previous models but seem crucial to help advance the discussion.

\section{Case density}

The vast differences that exist between endemic and non-endemic areas and

\footnotetext{
a Department of Preventive and Social Medicine, Jawaharlal Institute of Postgraduate Medical Education and Research, Pondicherry, India.

Correspondence to MB Soudarssanane (e-mail: drmybase@gmail.com).
} 
the impact this has on transmission dynamics within communities are rarely appreciated. The intensity of the infection pressure that exists in some endemic areas is evidenced by the frequency of disease caused by re-infection and/or multiple strain infection. ${ }^{5,6}$ Case density is a variable that describes the proximity of cases in space and time. From a transmission perspective, case density provides a measure of transmission overlap. Mathematical models using hypothetical scenarios assign a fixed number of secondary infections to each source case. By diagnosing and treating the source case it is assumed that a fixed number of secondary infections will be prevented. However, in endemic areas with high case densities the elimination of a single source case has limited impact on secondary infections among close contacts due to significant transmission overlap. Therefore, if we hope to develop mathematical models that are more robust and predictive of the situation in endemic areas, it seems relevant to adjust for case density. It should also be noted that, with transmission overlap, contacts are at risk of being infected multiple times by different source cases. The effect of repeated infections remains poorly understood, but multiple infectious challenges may "overwhelm" host immunity and predispose to the development of active disease.

\section{Transmission saturation}

Most source cases have fairly fixed circles of social interaction. This implies that once the majority of close contacts have been infected, the risk of infecting new people may decline even though the source case remains highly infectious. This phenomenon is referred to as transmission saturation. Transmission saturation illustrates the importance of early diagnosis and immediate institution of effective treatment. In this respect, the traditional focus on the most infectious sputum smear-positive cases, although clearly warranted, may not be the optimal strategy to limit the spread of disease within communities if it implies diagnostic delay beyond the point of transmission saturation. With delayed diagnosis, the transmission impact of ultimate treatment is greatly reduced. Current targets do not reflect the importance of limiting diagnostic delay and more sensitive diagnostic tools are required to achieve this.

There is a need to reconsider the accuracy and applicability of current mathematical models and to identify pragmatic ways of quantifying additional factors that may be at play in endemic areas. The incorporation of case density and transmission saturation in future mathematical models may assist the identification of more stringent, albeit more realistic, performance targets for global TB control.

Competing interests: None declared.

\section{BJ Marais ${ }^{\mathrm{a}}$ PD van Helden}

\section{References}

1. Dowdy DW, Chaisson RE. The persistence of tuberculosis in the age of DOTS: reassessing the effect of case detection. Bull World Health Organ 2009;87:296-304. PMID:19551238 doi:10.2471/BLT.08.054510

2. Resolution WHA 44.8. In: Forty-fourth World Health Assembly, Geneva, 6-16 May, 1991. Resolutions and Decisions. Geneva: World Health Organization;1991 (WHA44/1991/REC/1).

3. Styblo K, Bumgarner JR. Tuberculosis can be controlled with existing technologies: evidence. The Hague: Tuberculosis Surveillance Research Unit; 1991.

4. Marais BJ, Obihara CC, Warren RW, Schaaf HS, Gie RP, Donald PR. The burden of childhood tuberculosis: a public health perspective. Int J Tuberc Lung Dis 2005;9:1305-13. PMID:16466051

5. Van Rie A, Warren R, Richardson M, Victor TC, Gie RP, Enarson DA, et al. Exogenous reinfection as a cause of recurrent tuberculosis after curative treatment. N Engl J Med 1999; 341:1174-9. PMID:10519895 doi:10.1056/ NEJM199910143411602

6. Warren RM, Victor TC, Streicher EM, Richardson M, Beyers N, van Pittius NC, et al. Patients with active tuberculosis often have different strains in the same sputum specimen. Am J Respir Crit Care Med 2004;169:610-4. PMID:14701710 doi:10.1164/rccm.200305$7140 \mathrm{C}$

\section{Author reply}

Marais \& van Helden provide an important historical context for the role of mathematical modelling in formulating performance targets for tuberculosis (TB) control. Furthermore, they appropriately highlight that such models serve as simplifications of a far more complex reality, in which $M$. tuberculosis is transmitted in heterogeneous fashion. They mention two key factors - case density and transmission saturation - that contribute to such heterogeneity. However, there are many more, including nosocomial transmission clusters, ${ }^{1}$ strains of different fitness, ${ }^{2}$ social determinants of TB transmission ${ }^{3}$ and complex interactions with the HIV co-pandemic. ${ }^{4}$ Ultimately, no model can account for all potentially relevant aspects of TB transmission. Thus, we need simple models capable of distilling key components of transmission dynamics into clear messages. However, more complex models can be created to try to show us where - and to what degree - simple models may go wrong. Models exploring case density and transmission saturation could have an important role to play in this regard, and we welcome such efforts.

Ultimately, we must also remember that mathematical models are but one component of a broader TB research agenda that is sorely in need of expansion. ${ }^{5}$ While refining our models, we must not lose sight of the fact that approaches over the past 20 years have failed to stem the tide of ongoing TB transmission and that a broad-based, concerted effort - including an expanded research agenda, relentless improvements in case detection and development of better tools for TB diagnosis and treatment - will be required to meet current goals for TB control. Over the next 20 years, the value of TB mathematical models may be measured less by their ability to accurately describe the dynamics of TB transmission, and more by their power to galvanize support and inform appropriate policy.

Competing interests: None declared.

David W Dowdy ${ }^{\mathrm{C}}$ \& Richard E Chaisson $^{\mathrm{d}}$

\footnotetext{
a Department of Paediatrics and Child Health, Stellenbosch University, Tygerberg, South Africa. Correspondence to BJ Marais (e-mail: bjmarais@sun.ac.za).

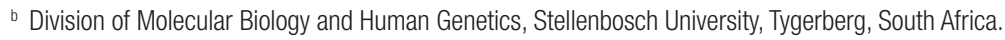

c University of California, San Francisco, CA United States of America.

d Johns Hopkins University, Baltimore, MD, USA. Correspondence to Richard E Chaisson (e-mail: rchaiss@jhmi.edu).
} 


\section{References}

1. Basu S, Friedland GH, Medlock J, Andrews JR, Shah NS, Gandhi NR, et al. Averting epidemics of extensively drug-resistant tuberculosis. Proc Natl Acad Sci USA 2009;106:7672-7.

PMID:19365076 doi:10.1073/ pnas.0812472106

2. Cohen T, Murray M. Modeling epidemics of multidrug-resistant M. tuberculosis of heterogeneous fitness. Nat Med 2004; 10:1117-21. PMID:15378056 doi:10.1038/ nm1110

3. Lönnroth K, Jaramillo E, Williams BG, Dye C, Raviglione M. Drivers of tuberculosis epidemics: the role of risk factors and social determinants. Soc Sci Med 2009;68:2240-6. PMID:19394122 doi:10.1016/j.socscimed.2009.03.041

4. Williams BG, Korenromp EL, Gouws E, Schmid GP, Auvert B, Dye C. HIV infection, antiretroviral therapy, and CD4+ cell count distributions in African populations. J Infect Dis 2006;194:1450-8. PMID:17054076 doi:10.1086/508206

5. Chaisson RE, Harrington M. How research can help control tuberculosis. Int J Tuberc Lung Dis 2009;13:558-68. PMID:19383187 